\title{
Methyl Methacrylate Synthesis: Thermodynamic Analysis for Oxidative Esterification of Methacrolein and Aldol Condensation of Methyl Acetate
}

Yanan Guan ${ }^{\mathrm{a}, \dagger}$, Hongqin Ma ${ }^{\mathrm{a}, \dagger}$, Wenyao Chen ${ }^{\mathrm{a},}{ }^{*}$, Maoshuai Li ${ }^{\mathrm{b}}$, Gang Qian ${ }^{\mathrm{a}}$, De Chen ${ }^{\mathrm{c}}$, Xinggui Zhou ${ }^{\mathrm{a}}$, Xuezhi Duan ${ }^{\mathrm{a},{ }^{*}}$

${ }^{a}$ State Key Laboratory of Chemical Engineering, East China University of Science and Technology, 130 Meilong Road, Shanghai 200237, China

${ }^{b}$ Key Laboratory for Green Chemical Technology of Ministry of Education, Collaborative Innovation Center of Chemical Science and Engineering, School of Chemical Engineering \& Technology, Tianjin University, Tianjin 300350, China

cDepartment of Chemical Engineering, Norwegian University of Science and Technology, N-7491 Trondheim, Norway

†These authors contributed equally to this work.

${ }^{*}$ To whom correspondence should be addressed. Tel.:+86-21-64250937; Email: wenyao.chen@ecust.edu.cn; xzduan@ecust.edu.cn. 
Table S1. $\Delta_{f} H_{298.15^{\theta}}, S_{298.15^{\theta}}$ and heat capacity of the reactants and products.

\begin{tabular}{lcccccc}
\hline \multirow{2}{*}{ Species } & $\Delta_{f} H_{298.15^{\theta}}$ & $S_{298.15^{\theta}}$ & \multicolumn{4}{c}{ Heat Capacity $c_{p}\left(\mathrm{~J} \cdot \mathrm{mol}^{-1} \cdot \mathrm{K}^{-1}\right)$} \\
\cline { 4 - 7 } & $\left(\mathrm{kJ} \cdot \mathrm{mol}^{-1}\right)$ & $\left(\mathrm{J} \cdot \mathrm{mol}^{-1} \cdot \mathrm{K}^{-1}\right)$ & $\mathrm{A}$ & $\mathrm{B} \times 10^{3}$ & $\mathrm{C} \times 10^{5}$ & $\mathrm{D} \times 10^{7}$ \\
\hline $\mathrm{O}_{2}$ & 0 & 205.2 & 29.53 & -8.90 & 3.81 & -0.33 \\
$\mathrm{MeOH}$ & -201.0 & 239.9 & 40.046 & -38.29 & 24.53 & -2.17 \\
$\mathrm{MAL}$ & -112.0 & 332.0 & 14.51 & 159.22 & 31.12 & -4.21 \\
$\mathrm{MMA}$ & -347.4 & 401.0 & -25.53 & 606.3 & -50.63 & 2.24 \\
$\mathrm{MAA}$ & -367.9 & 350.4 & -28.13 & 547.44 & -54.00 & 2.86 \\
$\mathrm{MF}$ & -357.4 & 285.3 & 5.79 & 250.72 & -17.52 & 0.61 \\
$\mathrm{H}$ & -241.8 & 188.8 & 33.93 & -8.42 & 3.00 & -0.33 \\
\hline
\end{tabular}


Table S2. $\Delta_{r} H_{T}{ }^{\theta}, \Delta_{r} S_{T}{ }^{\theta}, \Delta_{r} G_{T}{ }^{\theta}$, and $K_{p}$ for the reaction of MAL $(\mathrm{g})+1 / 2 \mathrm{O}_{2} \rightarrow$ MAA (g) at different temperatures.

\begin{tabular}{cccccc}
\hline $\mathrm{T}(\mathrm{K})$ & 303.15 & 313.15 & 323.15 & 333.15 & 343.15 \\
\hline$\Delta_{r} H_{T}^{\theta}\left(\mathrm{kJ} \cdot \mathrm{mol}^{-1}\right)$ & -255.94 & -255.94 & -255.93 & -255.92 & -255.91 \\
$\Delta_{r} S_{T}^{\theta}\left(\mathrm{J} \cdot \mathrm{mol}^{-1} \cdot \mathrm{K}^{-1}\right)$ & -84.19 & -84.18 & -84.15 & -84.12 & -84.09 \\
$\Delta_{r} G_{T}^{\theta}\left(\mathrm{kJ} \cdot \mathrm{mol}^{-1}\right)$ & -230.42 & -229.58 & -228.73 & -227.89 & -227.05 \\
$K^{\theta}$ & $5.06 \mathrm{E}+39$ & $1.97 \mathrm{E}+38$ & $9.43 \mathrm{E}+36$ & $5.40 \mathrm{E}+35$ & $3.66 \mathrm{E}+34$ \\
$\ln K^{\theta}$ & 91.42 & 88.18 & 85.14 & 82.28 & 79.58 \\
\hline & & & & & \\
\hline $\mathrm{T}(\mathrm{K})$ & 353.15 & 363.15 & 373.15 & 383.15 & 393.15 \\
\hline$\Delta_{r} H_{T}^{\theta}\left(\mathrm{kJ} \cdot \mathrm{mol}^{-1}\right)$ & -255.89 & -255.88 & -255.86 & -255.84 & -255.83 \\
$\Delta_{r} S_{T}^{\theta}\left(\mathrm{J} \cdot \mathrm{mol}^{-1} \cdot \mathrm{K}^{-1}\right)$ & -84.04 & -84.00 & -83.96 & -83.91 & -83.87 \\
$\Delta_{r} G_{T}^{\theta}\left(\mathrm{kJ} \cdot \mathrm{mol}^{-1}\right)$ & -226.21 & -225.37 & -224.53 & -223.69 & -222.85 \\
$K^{\theta}$ & $2.89 \mathrm{E}+33$ & $2.62 \mathrm{E}+32$ & $2.70 \mathrm{E}+31$ & $3.14 \mathrm{E}+30$ & $4.07 \mathrm{E}+29$ \\
$\ln K^{\theta}$ & 77.05 & 74.65 & 72.37 & 70.22 & 68.18 \\
\hline
\end{tabular}


Table S3. $\Delta_{r} H_{T}^{\theta}, \Delta_{r} S_{T}{ }^{\theta}, \Delta_{r} G_{T}{ }^{\theta}$, and $K_{p}$ for the reaction of MAA $(\mathrm{g})+\mathrm{MeOH}(\mathrm{g}) \rightarrow$ MMA $(\mathrm{g})+\mathrm{H}_{2} \mathrm{O}(\mathrm{g})$ at different temperatures.

\begin{tabular}{cccccc}
\hline $\mathrm{T}(\mathrm{K})$ & 303.15 & 313.15 & 323.15 & 333.15 & 343.15 \\
\hline$\Delta_{r} H_{T}^{\theta}\left(\mathrm{kJ} \cdot \mathrm{mol}^{-1}\right)$ & -20.17 & -20.07 & -19.98 & -19.88 & -19.78 \\
$\Delta_{r} S_{T}^{\theta}\left(\mathrm{J} \cdot \mathrm{mol}^{-1} \cdot \mathrm{K}^{-1}\right)$ & -0.35 & -0.03 & 0.27 & 0.57 & 0.86 \\
$\Delta_{r} G_{T}^{\theta}\left(\mathrm{kJ} \cdot \mathrm{mol}^{-1}\right)$ & -20.07 & -20.06 & -20.07 & -20.07 & -20.08 \\
$K^{\theta}$ & 2868.05 & 2222.56 & 1751.77 & 1402.10 & 1138.06 \\
$\ln K^{\theta}$ & 7.96 & 7.71 & 7.47 & 7.25 & 7.04 \\
\hline $\mathrm{T}(\mathrm{K})$ & & & & & \\
\hline$\Delta_{r} H_{T}^{\theta}\left(\mathrm{kJ} \cdot \mathrm{mol}^{-1}\right)$ & -19.68 & -19.59 & -19.49 & -19.39 & -19.29 \\
$\Delta_{r} S_{T}^{\theta}\left(\mathrm{J} \cdot \mathrm{mol}^{-1} \cdot \mathrm{K}^{-1}\right)$ & 1.14 & 1.42 & 1.68 & 1.94 & 2.19 \\
$\Delta_{r} G_{T}^{\theta}\left(\mathrm{kJ} \cdot \mathrm{mol}^{-1}\right)$ & -20.09 & -20.10 & -20.11 & -20.13 & -20.15 \\
$K^{\theta}$ & 935.64 & 778.28 & 654.37 & 555.65 & 476.13 \\
$\ln K^{\theta}$ & 6.84 & 6.66 & 6.48 & 6.32 & 6.17 \\
\hline
\end{tabular}


Table S4. $\Delta_{r} H_{T}{ }^{\theta}, \Delta_{r} S_{T}{ }^{\theta}, \Delta_{r} G_{T}{ }^{\theta}$, and $K_{p}$ for the reaction of MAL (g) $+\mathrm{MeOH}(\mathrm{g})+1 / 2$ $\mathrm{O}_{2}(\mathrm{~g}) \rightarrow \mathrm{MMA}(\mathrm{g})+\mathrm{H}_{2} \mathrm{O}(\mathrm{g})$ at different temperatures.

\begin{tabular}{cccccc}
\hline $\mathrm{T}(\mathrm{K})$ & 303.15 & 313.15 & 323.15 & 333.15 & 343.15 \\
\hline$\Delta_{r} H_{T}{ }^{\theta}\left(\mathrm{kJ} \cdot \mathrm{mol}^{-1}\right)$ & -276.11 & -276.01 & -275.91 & -275.80 & -275.69 \\
$\Delta_{r} S_{T}^{\theta}\left(\mathrm{J} \cdot \mathrm{mol}^{-1} \cdot \mathrm{K}^{-1}\right)$ & -84.54 & -84.21 & -83.88 & -83.55 & -83.23 \\
$\Delta_{r} G_{T}{ }^{\theta}\left(\mathrm{KJ} \cdot \mathrm{mol}^{-1}\right)$ & -250.48 & -249.64 & -248.80 & -247.96 & -247.13 \\
$K^{\theta}$ & $1.45 \mathrm{E}+43$ & $4.39 \mathrm{E}+41$ & $1.65 \mathrm{E}+40$ & $7.58 \mathrm{E}+38$ & $4.16 \mathrm{E}+37$ \\
$\ln K^{\theta}$ & 99.38 & 95.89 & 92.61 & 89.52 & 86.62 \\
\hline & & & & & \\
\hline $\mathrm{T}(\mathrm{K})$ & 353.15 & 363.15 & 373.15 & 383.15 & 393.15 \\
\hline$\Delta_{r} H_{T}{ }^{\theta}\left(\mathrm{kJ} \cdot \mathrm{mol}^{-1}\right)$ & -275.57 & -275.46 & -275.35 & -275.23 & -275.12 \\
$\Delta_{r} S_{T}^{\theta}\left(\mathrm{J} \cdot \mathrm{mol}^{-1} \cdot \mathrm{K}^{-1}\right)$ & -82.90 & -82.58 & -82.27 & -81.97 & -81.68 \\
$\Delta_{r} G_{T}^{\theta}\left(\mathrm{KJ} \cdot \mathrm{mol}^{-1}\right)$ & -246.30 & -245.47 & -244.65 & -243.82 & -243.01 \\
$K^{\theta}$ & $2.70 \mathrm{E}+36$ & $2.04 \mathrm{E}+35$ & $1.77 \mathrm{E}+34$ & $1.74 \mathrm{E}+33$ & $1.94 \mathrm{E}+32$ \\
$\ln K^{\theta}$ & 83.89 & 81.30 & 78.86 & 76.54 & 74.34 \\
\hline
\end{tabular}


Table S5. $\Delta_{r} H_{T}^{\theta}, \Delta_{r} S_{T}^{\theta}, \Delta_{r} G_{T}^{\theta}$, and $K_{p}$ for the reaction of $\mathrm{MeOH}(\mathrm{g})+1 / 2 \mathrm{O}_{2}(\mathrm{~g}) \rightarrow 1 / 2$ $\mathrm{MF}(\mathrm{g})+\mathrm{H}_{2} \mathrm{O}(\mathrm{g})$ at different temperatures.

\begin{tabular}{cccccc}
\hline $\mathrm{T}(\mathrm{K})$ & 303.15 & 313.15 & 323.15 & 333.15 & 343.15 \\
\hline$\Delta_{r} H_{T}^{\theta}\left(\mathrm{kJ} \cdot \mathrm{mol}^{-1}\right)$ & -219.47 & -219.40 & -219.33 & -219.26 & -219.18 \\
$\Delta_{r} S_{T}^{\theta}\left(\mathrm{J} \cdot \mathrm{mol}^{-1} \cdot \mathrm{K}^{-1}\right)$ & -10.94 & -10.72 & -10.50 & -10.28 & -10.06 \\
$\Delta_{r} G_{T}^{\theta}\left(\mathrm{kJ} \cdot \mathrm{mol}^{-1}\right)$ & -216.15 & -216.04 & -215.94 & -215.83 & -215.73 \\
$K^{\theta}$ & $1.76 \mathrm{E}+37$ & $1.09 \mathrm{E}+36$ & $8.05 \mathrm{E}+34$ & $6.94 \mathrm{E}+33$ & $6.92 \mathrm{E}+32$ \\
$\ln K^{\theta}$ & 85.76 & 82.98 & 80.37 & 77.92 & 75.62 \\
\hline & & & & & \\
\hline$\Delta_{r} H_{T}^{\theta}\left(\mathrm{kJ} \cdot \mathrm{mol}^{-1}\right)$ & -219.11 & -219.04 & -218.96 & -218.89 & -218.81 \\
$\Delta_{r} S_{T}^{\theta}\left(\mathrm{J} \cdot \mathrm{mol}^{-1} \cdot \mathrm{K}^{-1}\right)$ & -9.85 & -9.65 & -9.44 & -9.24 & -9.05 \\
$\Delta_{r} G_{T}^{\theta}\left(\mathrm{kJ} \cdot \mathrm{mol}^{-1}\right)$ & -215.63 & -215.53 & -215.44 & -215.34 & -215.25 \\
$K^{\theta}$ & $7.86 \mathrm{E}+31$ & $1.01 \mathrm{E}+31$ & $1.44 \mathrm{E}+30$ & $2.28 \mathrm{E}+29$ & $3.98 \mathrm{E}+28$ \\
$\ln K^{\theta}$ & 73.44 & 71.39 & 69.44 & 67.60 & 65.85 \\
\hline
\end{tabular}


Table S6. $\Delta_{f} H_{298.15^{\theta}}, S_{298.15^{\theta}}$ and heat capacity of the reactants and products.

\begin{tabular}{|c|c|c|c|c|c|c|}
\hline \multirow{2}{*}{ Species } & \multirow{2}{*}{$\begin{array}{l}\Delta_{f} H_{298.15^{\theta}} \\
\left(\mathrm{kJ} \cdot \mathrm{mol}^{-1}\right)\end{array}$} & \multirow{2}{*}{$\begin{array}{c}S_{298.15}{ }^{\theta} \\
\left(\mathrm{J} \cdot \mathrm{mol}^{-1} \cdot \mathrm{K}^{-1}\right)\end{array}$} & \multicolumn{4}{|c|}{ Heat Capacity $c_{p}\left(\mathrm{~J} \cdot \mathrm{mol}^{-1} \cdot \mathrm{K}^{-1}\right)$} \\
\hline & & & A & $\mathrm{B} \times 10^{3}$ & $\mathrm{C} \times 10^{5}$ & $\mathrm{D} \times 10^{7}$ \\
\hline $\mathrm{MeOH}$ & -201.0 & 239.9 & 40.046 & -38.29 & 24.53 & -2.17 \\
\hline $\mathrm{MeOAC}$ & -413.3 & 324.4 & -22.29 & 482.75 & -46.63 & 2.33 \\
\hline $\mathrm{H}_{2}$ & 0 & 188.8 & 25.40 & 20.18 & -3.85 & 0.32 \\
\hline MMA & -347.4 & 401.0 & -25.53 & 606.28 & -50.63 & 2.24 \\
\hline MA & -333 & 365.6 & 1.22 & 406.19 & -28.53 & 0.97 \\
\hline MP & -427.5 & 359.6 & -131.95 & 1376.70 & -247.90 & 22.38 \\
\hline FY & -108.6 & 218.8 & 34.43 & -29.78 & 15.10 & -1.27 \\
\hline $\mathrm{H}_{2} \mathrm{O}$ & -241.8 & 188.8 & 33.93 & -8.42 & 3.00 & -0.33 \\
\hline
\end{tabular}


Table S7. $\Delta_{r} H_{T}^{\theta}, \Delta_{r} S_{T}^{\theta}, \Delta_{r} G_{T}^{\theta}$, and $K_{p}$ for the reaction of MeOAC $(\mathrm{g})+\mathrm{FY}(\mathrm{g}) \rightarrow \mathrm{MA}$ $(\mathrm{g})+\mathrm{H}_{2} \mathrm{O}(\mathrm{g})$ at different temperature.

\begin{tabular}{ccccc}
\hline $\mathrm{T}(\mathrm{K})$ & 373.15 & 473.15 & 573.15 & 673.15 \\
\hline$\Delta_{r} H_{T}^{\theta}\left(\mathrm{kJ} \cdot \mathrm{mol}^{-1}\right)$ & -52.13 & -51.29 & -50.62 & -50.09 \\
$\Delta_{r} S_{T}^{\theta}\left(\mathrm{J} \cdot \mathrm{mol}^{-1} \cdot \mathrm{K}^{-1}\right)$ & 13.51 & 15.50 & 16.79 & 17.66 \\
$\Delta_{r} G_{T}^{\theta}\left({\left.\mathrm{kJ} \cdot \mathrm{mol}^{-1}\right)}^{-57.17}\right.$ & -58.63 & -60.25 & -61.97 \\
$K^{\theta}$ & $1.01 \mathrm{E}+09$ & $2.97 \mathrm{E}+06$ & $3.10 \mathrm{E}+05$ & $6.44 \mathrm{E}+04$ \\
$\ln K^{\theta}$ & 18.43 & 14.90 & 12.64 & $11 . .07$ \\
\hline & & & & \\
\hline $\mathrm{T}(\mathrm{K})$ & 873.15 & 973.15 & 1073.15 & 1173.15 \\
\hline$\Delta_{r} H_{T}^{\theta}\left(\mathrm{kJ} \cdot \mathrm{mol}^{-1}\right)$ & -49.37 & -49.21 & -49.22 & -49.47 \\
$\Delta_{r} S_{T}^{\theta}\left(\mathrm{J} \cdot \mathrm{mol}^{-1} \cdot \mathrm{K}^{-1}\right)$ & 18.60 & 18.78 & 18.77 & 18.55 \\
$\Delta_{r} G_{T}^{\theta}\left(\mathrm{kJ} \cdot \mathrm{mol}^{-1}\right)$ & -65.62 & -67.49 & -69.37 & -71.23 \\
$K^{\theta}$ & $8.42 \mathrm{E}+03$ & $4.19 \mathrm{E}+03$ & $2.38 \mathrm{E}+03$ & $1.49 \mathrm{E}+03$ \\
$\ln K^{\theta}$ & 9.04 & 8.34 & 7.77 & 7.30 \\
\hline
\end{tabular}


Table S8. $\Delta_{r} H_{T}{ }^{\theta}, \Delta_{r} S_{T}^{\theta}, \Delta_{r} G_{T}{ }^{\theta}$, and $K_{p}$ for the reaction of MA $(\mathrm{g})+\mathrm{H}_{2}(\mathrm{~g}) \rightarrow \mathrm{MP}(\mathrm{g})$ at different temperature.

\begin{tabular}{ccccc}
\hline $\mathrm{T}(\mathrm{K})$ & 373.15 & 473.15 & 573.15 & 673.15 \\
\hline$\Delta_{r} H_{T}^{\theta}\left(\mathrm{kJ} \cdot \mathrm{mol}^{-1}\right)$ & -95.43 & -96.09 & -96.61 & -97.14 \\
$\Delta_{r} S_{T}^{\theta}\left(\mathrm{J} \cdot \mathrm{mol}^{-1} \cdot \mathrm{K}^{-1}\right)$ & -139.51 & -141.11 & -142.10 & -142.95 \\
$\Delta_{r} G_{T}^{\theta}\left(\mathrm{kJ} \cdot \mathrm{mol}^{-1}\right)$ & -43.37 & -29.33 & -15.17 & -0.91 \\
$K^{\theta}$ & $1.18 \mathrm{E}+06$ & $1 . .73 \mathrm{E}+03$ & $2.41 \mathrm{E}+01$ & $1.18 \mathrm{E}+00$ \\
$\ln K^{\theta}$ & 13.98 & 7.46 & 3.18 & 0.16 \\
\hline & & & & \\
\hline $\mathrm{T}(\mathrm{K})$ & 873.15 & 973.15 & 1073.15 & 1173.15 \\
\hline$\Delta_{r} H_{T}^{\theta}\left(\mathrm{kJ} \cdot \mathrm{mol}^{-1}\right)$ & -97.84 & -97.85 & -97.90 & -98.72 \\
$\Delta_{r} S_{T}^{\theta}\left(\mathrm{J} \cdot \mathrm{mol}^{-1} \cdot \mathrm{K}^{-1}\right)$ & -143.89 & -143.90 & -143.94 & -144.67 \\
$\Delta_{r} G_{T}^{\theta}\left(\mathrm{kJ} \cdot \mathrm{mol}^{-1}\right)$ & 27.79 & 42.18 & 56.57 & 70.99 \\
$K^{\theta}$ & $2.17 \mathrm{E}-02$ & $5.44 \mathrm{E}-03$ & $1.76 \mathrm{E}-03$ & $6.90 \mathrm{E}-04$ \\
$\ln K^{\theta}$ & -3.83 & -5.21 & -6.34 & -7.28 \\
\hline
\end{tabular}


Table S9. $\Delta_{r} H_{T}^{\theta}, \Delta_{r} S_{T}^{\theta}, \Delta_{r} G_{T}{ }^{\theta}$, and $K_{p}$ for the reaction of MP $(\mathrm{g})+\mathrm{FY}(\mathrm{g}) \rightarrow \mathrm{MMA}(\mathrm{g})$ $+\mathrm{H}_{2} \mathrm{O}(\mathrm{g})$ at different temperature.

\begin{tabular}{ccccc}
\hline $\mathrm{T}(\mathrm{K})$ & 373.15 & 473.15 & 573.15 & 673.15 \\
\hline$\Delta_{r} H_{T}^{\theta}\left(\mathrm{kJ} \cdot \mathrm{mol}^{-1}\right)$ & -53.09 & -53.23 & -53.20 & -52.90 \\
$\Delta_{r} S_{T}^{\theta}\left(\mathrm{J} \cdot \mathrm{mol}^{-1} \cdot \mathrm{K}^{-1}\right)$ & 11.44 & 11.12 & 11.16 & 11.49 \\
$\Delta_{r} G_{T}^{\theta}\left(\mathrm{kJ} \cdot \mathrm{mol}^{-1}\right)$ & -57.36 & -58.49 & -59.60 & -60.73 \\
$K^{\theta}$ & $1.07 \mathrm{E}+08$ & $2.86 \mathrm{E}+06$ & $2.70 \mathrm{E}+05$ & $5.16 \mathrm{E}+04$ \\
$\ln K^{\theta}$ & 18.49 & 14.87 & 12.51 & 10.85 \\
\hline & & & & \\
\hline $\mathrm{T}(\mathrm{K})$ & 873.15 & 973.15 & 1073.15 & 1173.15 \\
\hline$\Delta_{r} H_{T}^{\theta}\left(\mathrm{kJ} \cdot \mathrm{mol}^{-1}\right)$ & -52.65 & -52.72 & -52.68 & -51.78 \\
$\Delta_{r} S_{T}^{\theta}\left(\mathrm{J} \cdot \mathrm{mol}^{-1} \cdot \mathrm{K}^{-1}\right)$ & 11.95 & 11.88 & 11.91 & 12.73 \\
$\Delta_{r} G_{T}^{\theta}\left(\mathrm{kJ} \cdot \mathrm{mol}^{-1}\right)$ & -63.09 & -64.28 & -65.47 & -66.69 \\
$K^{\theta}$ & $5.95 \mathrm{E}+03$ & $2.82 \mathrm{E}+03$ & $1.54 \mathrm{E}+03$ & $9.32 \mathrm{E}+02$ \\
$\ln K^{\theta}$ & 8.69 & 7.94 & 7.34 & 6.84 \\
\hline
\end{tabular}


Table S10. $\Delta_{r} H_{T}{ }^{\theta}, \Delta_{r} S_{T}{ }^{\theta}, \Delta_{r} G_{T}{ }^{\theta}$, and $K_{p}$ for the reaction of $\mathrm{MeOH}(\mathrm{g}) \rightarrow \mathrm{FY}(\mathrm{g})+\mathrm{H}_{2}$ (g) at different temperature.

\begin{tabular}{ccccc}
\hline $\mathrm{T}(\mathrm{K})$ & 373.15 & 473.15 & 573.15 & 673.15 \\
\hline$\Delta_{r} H_{T}^{\theta}\left(\mathrm{kJ} \cdot \mathrm{mol}^{-1}\right)$ & 94.00 & 95.93 & 97.50 & 98.54 \\
$\Delta_{r} S_{T}^{\theta}\left(\mathrm{J} \cdot \mathrm{mol}^{-1} \cdot \mathrm{K}^{-1}\right)$ & 114.40 & 119.00 & 122.01 & 123.69 \\
$\Delta_{r} G_{T}^{\theta}\left(\mathrm{kJ} \cdot \mathrm{mol}^{-1}\right)$ & 51.31 & 39.63 & 27.57 & 15.27 \\
$K^{\theta}$ & $6.56 \mathrm{E}-08$ & $4.22 \mathrm{E}-05$ & $3.07 \mathrm{E}-03$ & $6.53 \mathrm{E}-02$ \\
$\ln K^{\theta}$ & -16.54 & -10.07 & -5.78 & -2.73 \\
\hline & & & & \\
\hline $\mathrm{T}(\mathrm{K})$ & 873.15 & 973.15 & 1073.15 & 1173.15 \\
\hline$\Delta_{r} H_{T}^{\theta}\left(\mathrm{kJ} \cdot \mathrm{mol}^{-1}\right)$ & 98.26 & 96.46 & 93.16 & 87.98 \\
$\Delta_{r} S_{T}^{\theta}\left(\mathrm{J} \cdot \mathrm{mol}^{-1} \cdot \mathrm{K}^{-1}\right)$ & 123.44 & 121.50 & 118.28 & 113.68 \\
$\Delta_{r} G_{T}^{\theta}\left(\mathrm{kJ} \cdot \mathrm{mol}^{-1}\right)$ & -9.52 & -21.78 & -33.78 & -45.39 \\
$K^{\theta}$ & $3.71 \mathrm{E}+00$ & $1.48 \mathrm{E}+01$ & $4.41 \mathrm{E}+01$ & $1.05 \mathrm{E}+02$ \\
$\ln K^{\theta}$ & 1.31 & 2.69 & 3.79 & 4.65 \\
\hline
\end{tabular}


Table S11. $\Delta_{r} H_{T}{ }^{\theta}, \Delta_{r} S_{T}{ }^{\theta}, \Delta_{r} G_{T}{ }^{\theta}$, and $K_{p}$ for the reaction of MeOAC (g) $+2 \mathrm{MeOH}(\mathrm{g})$

$\rightarrow$ MMA $(\mathrm{g})+\mathrm{H}_{2}(\mathrm{~g})+2 \mathrm{H}_{2} \mathrm{O}(\mathrm{g})$ at different temperature.

\begin{tabular}{ccccc}
\hline $\mathrm{T}(\mathrm{K})$ & 373.15 & 473.15 & 573.15 & 673.15 \\
\hline$\Delta_{r} H_{T}^{\theta}\left(\mathrm{kJ} \cdot \mathrm{mol}^{-1}\right)$ & -12.64 & -8.75 & -5.44 & -3.15 \\
$\Delta_{r} S_{T}^{\theta}\left(\mathrm{J} \cdot \mathrm{mol}^{-1} \cdot \mathrm{K}^{-1}\right)$ & 114.24 & 123.52 & 129.88 & 133.59 \\
$\Delta_{r} G_{T}^{\theta}\left(\mathrm{kJ} \cdot \mathrm{mol}^{-1}\right)$ & -55.27 & -67.19 & -79.88 & -93.07 \\
$K^{\theta}$ & $5.47 \mathrm{E}+07$ & $2.62 \mathrm{E}+07$ & $1.91 \mathrm{E}+07$ & $1.67 \mathrm{E}+077$ \\
$\ln K^{\theta}$ & 17.82 & 17.08 & 16.76 & 16.63 \\
\hline & & & & \\
\hline $\mathrm{T}(\mathrm{K})$ & 873.15 & 973.15 & 1073.15 & 1173.15 \\
\hline$\Delta_{r} H_{T}^{\theta}\left(\mathrm{kJ} \cdot \mathrm{mol}^{-1}\right)$ & -3.35 & -6.86 & -13.49 & -23.99 \\
$\Delta_{r} S_{T}^{\theta}\left(\mathrm{J} \cdot \mathrm{mol}^{-1} \cdot \mathrm{K}^{-1}\right)$ & 133.54 & 129.76 & 123.31 & 113.98 \\
$\Delta_{r} G_{T}^{\theta}\left(\mathrm{kJ}^{\prime} \cdot \mathrm{mol}^{-1}\right)$ & -119.95 & -133.14 & -145.82 & -157.71 \\
$K^{\theta}$ & $1.50 \mathrm{E}+07$ & $1.40 \mathrm{E}+07$ & $1.25 \mathrm{E}+07$ & $1.05 \mathrm{E}+07$ \\
$\ln K^{\theta}$ & 16.52 & 16.46 & 16.34 & 16.17 \\
\hline
\end{tabular}

